\title{
Isothermal programming of triple shape memory
}

\author{
Jing Zhou, Qiaoxi Li, Sara A. Turner, Valerie Sheares Ashby, and Sergei S. Sheiko* \\ Department of Chemistry, University of North Carolina at Chapel Hill, NC
}

$27599-3290$

\begin{abstract}
Here we present a new strategy for enabling triple shape memory (TSM), in which different shapes may be programmed at one constant fixation temperature during isothermal crystallization of a poly(octylene adipate) elastomer. Unlike traditional TSM programming techniques that utilize separate thermal transitions to encode each distinct shape, here we report that multiple shapes may be encoded independently at different time stages of the same isothermal crystallization process, and then recovered sequentially at different temperatures upon heating. As such, the new method extends TSM capabilities to conventional semicrystalline elastomers that exhibit a single and narrow thermal transition. Unexpectedly, shape fixation does not depend on crystallinity acquired prior to sample deformation, which suggests that different shapes are secured by an individual crystalline scaffold with minor contribution of the pre-existing crystals. To that end, the isothermal protocol for TSM programming was utilized to demonstrate a "one-way reversible" shape memory transformation controlled by fixation time.
\end{abstract}




\section{INTRODUCTION}

Among stimuli-responsive materials, shape memory polymers are unique in their ability to undergo programmable transformations from one complex shape to another [1-8]. Conventional shape memory materials typically undergo a single shape change from a temporary shape to a permanent shape, while triple or multiple shape memory materials have been designed to enable transitions between three and more shapes [9-18]. A variety of strategies have been developed for encoding triple shape memory (TSM). One strategy requires the use of a two-phase heterogeneous material such as block-copolymers and composites, in which each phase has a distinct crystallization and/or glass transition temperature [9-13]. In this way, a different shape can be fixed at each distinct thermal transition. Another approach to the realization of TSM is based on the temperature memory effect (TME), which suggests that materials have memory of the temperature at which the shape was fixed [14-18]. This approach requires polymers with a broad crystallization or glass transition to allow programming of unique shapes at different temperatures within the same transition region [14].

In each of these cases, particular materials have to be designed to allow TSM programming at different temperatures. For many applications, however, it would be advantageous to program different shapes under isothermal conditions. This would enable utilization of more conventional materials, such as those with a single and narrow thermal transition. Here we report the isothermal programming of TSM by controlling the crystallization rate of a semicrystalline elastomer. The concept is universal and can be applied to most semicrystalline shape memory elastomers. Figure 1 shows a general protocol for isothermal shape programming. Similar to conventional protocols, a network of chemical crosslinks is responsible for defining a permanent equilibrium shape, and a crystallization process provides a crystalline scaffold for fixation of a temporary shape. By using crystallites formed at different times, we can potentially encode many different shapes during an isothermal 
crystallization process. This strategy is analogous to a chemical aging study by Tobolsky et al, in which two populations of network strands were introduced to be in equilibrium with deformed and original shapes, respectively [19].

The new shape-programming protocol can extend TSM to temperature sensitive and delicate applications such as minimally invasive surgery $[1,18]$. Encoding distinct shapes at different stages of an isothermal crystallization process offers a new method for studying relationships between the microscopic structure and the macroscopic properties, such as shape and modulus, of semicrystalline elastomers. This approach may shed light on the mechanisms of correlation between hierarchical polymer crystallization and shape memory actuation [20-23]. To that end, we utilized a programming protocol for one-way reversible shape transition [8, 24], and demonstrated that distinct contributions from different sections of the same crystalline scaffold can result in two oppositely-directed shape transformations. 
(a)<smiles>CCC(COC(=O)CCS)OC(=O)CCS</smiles>

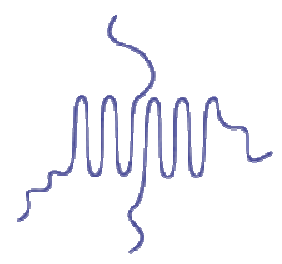

Shape 2
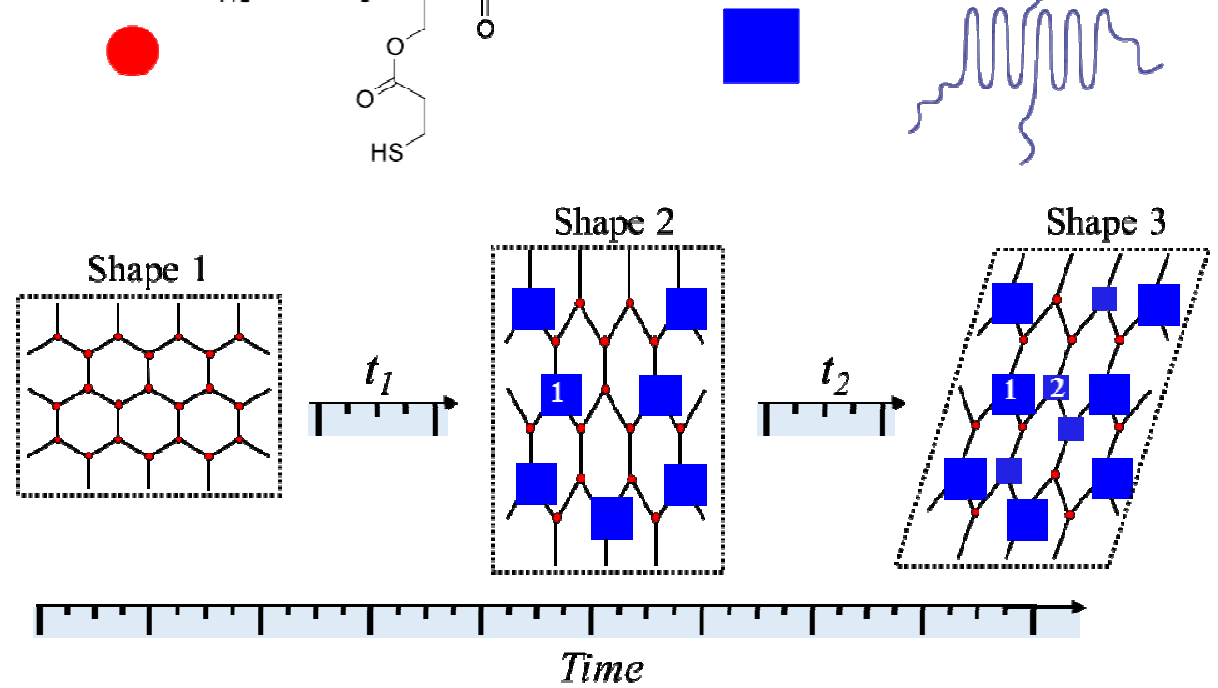

(b)

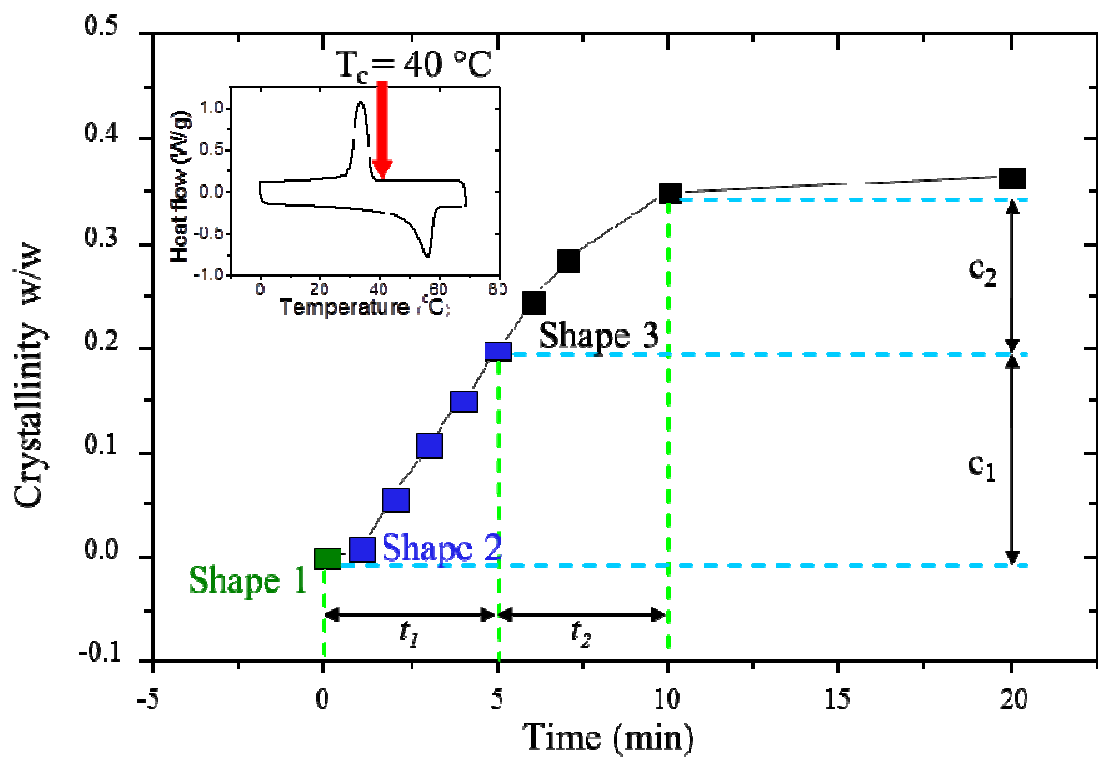

Figure 1: Memorization of multiple shapes with isothermal crystallization. (a) Crystals formed at different time intervals can be used to memorize different shapes. Red dots represent the permanent chemical crosslinks of trimethylolpropane tris(3-mercaptopropionate) forming the polymer network (black lines). Blue squares represents crystals formed at different stages in isothermal crystallization: 1 - early stages and 2 - later stages of crystallization (b) DSC and WAXS measurements show that the crystallinity of semicrystalline elastomer POA increases with time during isothermal crystallization at $40{ }^{\circ} \mathrm{C}$ (see also Supplementary Figure S2). The sequential nucleation and growth of crystallites allows fixation of distinct shapes at different stages of an isothermal crystallization process. 


\section{EXPERIMENTAL METHODS}

Materials. Adipic acid (Alfa Aesar, 99\%), 1,8-octandiol (Goldbio), 2,2-diethoxyacetophenone (Acros, 98\%), tin(II) 2-ethylhexanoate (Sigma Aldrich, 95\%), scandium(III) triflate (Sigma Aldrich, 99\%), 2-isocyanatoethyl methacrylate (TCI America, 98\%), trimethylolpropane tris(3-mercaptopropionate) (Sigma Aldrich, 95\%) and Novozym 435 (Sigma Aldrich) were used as received. Solvents were purchased from VWR International.

Poly(octylene adipate) (POA) (Figure 2a) We recently reported the synthesis of POA $[8,24]$. Endfunctionalization of POA with 2-isocyanatoethyl methacrylate is reported elsewhere [25].

POA thermoset elastomers. POA ( $2 \mathrm{~g}$ ) was dried under vacuum for 24 hours at 45 ${ }^{\circ} \mathrm{C}$ before dissolution in $1 \mathrm{~mL} \mathrm{CHCl}_{3}$. The crosslinker - trimethylolpropane tris(3-mercaptopropionate) $(80 \mu \mathrm{L})$ and initiator - 2,2-diethoxyacetophenone $(35 \mu \mathrm{L})$ were then added, and after mixing the solution was poured into the desired mold and irradiated for 10 min under UV light. The crosslinked sample was then dried under vacuum at $70{ }^{\circ} \mathrm{C}$ for $\geq 24$ hours to remove any remaining solvent. The gel fraction was measured after Soxhlet extraction in $\mathrm{CHCl}_{3}$ for $24 \mathrm{~h}$ and subsequent vacuum drying. The molar crosslinking density was calculated as $v=E /(3 R T)$, where $E$ is the tensile storage modulus measured by DMA at strain $\varepsilon=0.1 \%$, frequency $f=1 \mathrm{~Hz}$, and temperature $T=80^{\circ} \mathrm{C}$.

Differential scanning calorimetry. DSC 220 from Seiko Instruments, Inc. was used to measure crystallinity changes during the isothermal crystallization process. A sample was first completely melted at $70{ }^{\circ} \mathrm{C}$, and then rapidly cooled to the targeted crystallization temperature with a speed of $60^{\circ} \mathrm{C} / \mathrm{min}$. The crystallization temperature was kept constant for a given time before heating at $10^{\circ} \mathrm{C} / \mathrm{min}$. The crystallinity was calculated as $\phi=\Delta H_{m} / \Delta H_{m}^{0}\left(\Delta H_{m}^{0}=150 \mathrm{~J} / \mathrm{g}\right)$ from integrating the melting transition. 
Dynamic mechanical analysis. RSA-G2 Dynamic Mechanical Analyzer from TA Instruments was employed both for fixation and recovery experiments. Dogbone-shaped samples $(7 \mathrm{~mm} \times 2.5 \mathrm{~mm} \times 1 \mathrm{~mm})$ were utilized, and isothermal shape fixation was performed in two steps. Initially, the sample was kept at an initial length of $l=l_{0}$ for time $t_{1}$, which was followed by its stretching to a programmed strain $\varepsilon_{p}=\left(l_{p}-l_{0}\right) / l_{0}$ and isothermal annealing at $\mathrm{T}=40{ }^{\circ} \mathrm{C}$ for time $t_{2}$. When the external stress was released, the sample was free to contract to a final strain $\varepsilon_{f}=$ $\left(l_{f}-l_{0}\right) / l_{0}$, where $l_{f}$ is the sample length after stress release. The fixity ratio is calculated as a ratio of the free and programmed strains $F=\varepsilon_{f} / \varepsilon_{p}$. The total crystallization time $t_{1}+t_{2}=10 \mathrm{~min}$ was maintained throughout all recovery tests in order to maximize crystallinity. After $10 \mathrm{~min}$ at the crystallization temperature, the sample was heated at a rate of $5^{\circ} \mathrm{C} / \mathrm{min}$ to $70{ }^{\circ} \mathrm{C}$ at zero external force.

Triple shape programming. The angle changes of hairpin samples of POA were used for the quantitative analysis of shape change. The small thickness of the sample $(\sim 0.8 \mathrm{~mm})$ allowed quick heating or quenching of sample, and insured uniform crystallization and melting. We previously reported a type of TSM behavior in which the third shape is programmed to appear similar to the original shape, which we refer to as one-way reversibility [8,24]. In this paper, we applied the same programming protocol. The initial unbent sample was heated to $70^{\circ} \mathrm{C}$, bent $180^{\circ}$ to a hairpin shape, and then quenched to the crystallization temperature of $40{ }^{\circ} \mathrm{C}$. The temperature was chosen to be slightly higher than $T_{c}$ to ensure a slower crystallization rate and thus allow ample time for programming. The crystallization time for the first fixation was varied as $t_{1}$. The sample was then unbent back to a straight ribbon and crystallization continued $\left(t_{2}\right)$, until a total crystallization time of 10 min was reached.

Atomic force microscopy. Topographic images of a crosslinked spin-coated elastomer surface were collected using a multimode Atomic Force Microscope with a NanoScope V controller in PeakForce QNM mode (Bruker). We used silicon cantilevers with a resonance frequency of $\sim 70 \mathrm{kHz}$ and a spring constant of $\sim 0.4 \mathrm{~N} / \mathrm{m}$. 
Wide angle X-ray scattering (WAXS). Measurements of WAXS spectra of shape memory elastomers were performed at the NSLS X9 beamline and on the CFN Bruker Nanostar WAXS instrument at Brookhaven National Laboratory using area detectors.

(a)<smiles>C=C(C)C(=O)OCCNC(=O)OCCOC(=O)CCCCC(=O)OCCOC(=O)NCCOC(=O)C(=C)C</smiles>
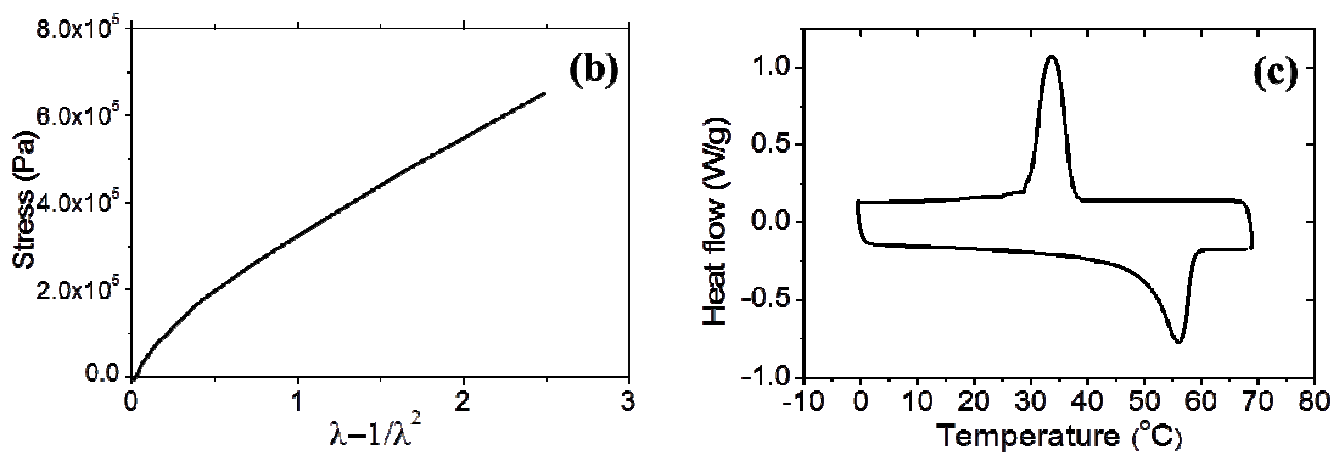

Figure 2: Semicrystalline shape memory materials. Chemical structure of (a) methacrylate-terminated poly(octylene adipate) (POA) (b) Engineering stress as a function of elongation of POA measured at $\mathrm{T}=80^{\circ} \mathrm{C}$.. (c) DSC traces of POA, with heating and cooling rate of $5{ }^{\circ} \mathrm{C} / \mathrm{min}$.

Table 1: Material properties of shape memory polymers

\begin{tabular}{|l|c|c|c|c|c|c|c|c|}
\hline$\#$ & Polymer name & $\mathrm{DP}_{\mathrm{n}}{ }^{\mathrm{b}}$ & $\begin{array}{c}\mathrm{T}_{\mathrm{m}}{ }^{\mathrm{c}} \\
{ }^{\circ} \mathrm{C}\end{array}$ & $\begin{array}{c}\mathrm{T}_{\mathrm{c}}{ }^{\mathrm{c}} \\
{ }^{\circ} \mathrm{C}\end{array}$ & $\begin{array}{c}\Delta \mathrm{H}_{\mathrm{m}}{ }^{\mathrm{c}} \\
\mathrm{J} / \mathrm{g}\end{array}$ & $\begin{array}{c}\mathrm{E}^{\mathrm{d}} \\
\mathrm{MPa}\end{array}$ & $\begin{array}{c}\mathrm{v}^{\mathrm{e}} \\
\mathrm{mol} / \mathrm{m}^{3}\end{array}$ & $\phi^{\mathrm{f}}$ \\
\hline 1 & $\begin{array}{c}\text { Thiol crosslinked } \\
\text { POA polymer }\end{array}$ & 14.7 & 56 & 34 & 62 & 2.4 & 280 & 0.94 \\
\hline
\end{tabular}

(a) Chemical structures in Figure 2; (b) Number average degree of polymerization determined by proton NMR; (c) Temperatures of melting and crystallization, and enthalpy of melting measured by DSC, second heat, at $5{ }^{\circ} \mathrm{C} / \mathrm{min}$; (d) Tensile storage modulus measured by DMA at $T=80{ }^{\circ} \mathrm{C}$ and $f=1 \mathrm{~Hz}$; (e) Apparent crosslinking density calculated as $v=E /(3 R T)$; (f) Gel fraction is calculated as $\phi=m_{d} / m_{0}$, where $m_{0}$ is the initial mass and $m_{d}$ is dry residue mass after Soxhlet extraction $\left(\mathrm{CHCl}_{3}\right.$ for $\left.24 \mathrm{~h}\right)$ and drying in vacuum oven for $24 \mathrm{~h}$. 


\section{RESULTS AND DISCUSSION}

\section{Material characterization}

Material properties of the studied elastomer are summarized in Table 1. In addition, for calibration purposes, we studied other POA compositions that are outlined in Supplementary Table S1. Mechanical properties (modulus and elongation at break) of the elastomers depend on the fraction of the crosslinker with respect to the POA oligomers. Figure $2 b$ shows a stress-strain curve for an elastomer prepared from a 1 $\mathrm{mol} / \mathrm{mol}$ crosslinker/POA composition (1:1 ratio between moles of functional groups in crosslinker and prepolymer), which exhibits significant extensibility. In shape programming experiments, we kept strain below $50 \%$ to minimize the strain effect on crystallization kinetics. The thermal behavior was analyzed with DSC (Figure 2c, Supplementary Figure S1), showing typical crystallization and melting of a semi-crystalline polymer. Similar to the other POA based copolymers $[8,24,25]$, the crystallization and melting points are close to typical physiological temperatures enabling potential medical applications. The kinetics of crystallization of POA was monitored independently by DSC and WAXS showing consistent results. (Figure 1b, Supplementary Figure S2)

\section{Shape Fixation}

As shown in Figure 1b, isothermal annealing of a thiol crosslinked POA polymer at $40{ }^{\circ} \mathrm{C}$ results in a continuous increase of crystallinity leveling off at $36 \mathrm{w} / \mathrm{w} \%$. During shape programming, the crystalline phase was used to secure a temporary shape. As shown previously, however, shape fixation does not require full crystallization [8]. For our samples, ca. $25 \%$ crystallinity is sufficient to secure the temporary shape (Figure 3c), and any additional crystals formed at later stages of crystallization are redundant in a conventional shape-programming protocol. Yet, these "redundant" crystals are potentially available for encoding additional shapes. To 
investigate this possibility, we utilized DMA and DSC to quantify shape fixation by crystals formed at later stages of isothermal crystallization.

Figure 3a outlines a protocol for shape fixation upon uniaxial extension, which was applied at various times during isothermal crystallization of POA at $\mathrm{T}=40{ }^{\circ} \mathrm{C}$. We have partitioned the crystallization process in two time intervals: (i) isothermal crystallization of the original length $l=l_{0}(\varepsilon=0 \%)$ for time interval $t_{1}$ and (ii) isothermal crystallization under uniaxial extension $\varepsilon_{p}=\left(l_{p}-l_{0}\right) / l_{0}=15 \%$ for time interval $t_{2}$. In order to measure shape fixity, the external stress was released after $t_{2}$, allowing the POA elastomer to contract to $\varepsilon_{f}=\left(l_{f}-l_{0}\right) / l_{0}$, where $l_{f}$ is the sample length after the stress release. The fixity parameter is calculated as a ratio of the free and programmed strains $F=\varepsilon_{f} / \varepsilon_{p}$. As shown in Figure 3b, the sample was able to achieve a high fixity of $F>80 \%$ even for stretching after $t_{1}=5 \mathrm{~min}$ of crystallization when $>50 \%$ of the total crystallinity was reached (Figure 1b). Availability of this idle crystallinity suggests that more than one shape can be encoded during isothermal crystallization by utilizing different fractions of crystallites for fixation of different shapes. Utilizing the crystallization kinetics data shown in Figure $1 \mathrm{~b}$, the crystallization time was converted to degree of crystallinity assuming a small effect of strain-induced crystallization for 15\% deformation (Supplementary Figure S3). Surprisingly, if we plot the fixity ratio with respect to weight fraction of the crystalline phase formed during $t_{2}$, all fixation curves recorded for different pre-stretching crystallization intervals $\left(t_{1}\right)$ collapsed on to one universal curve (Figure 3c). This suggests that new crystals were able to encode a distinct shape independently of the presence of pre-existing crystallites. 


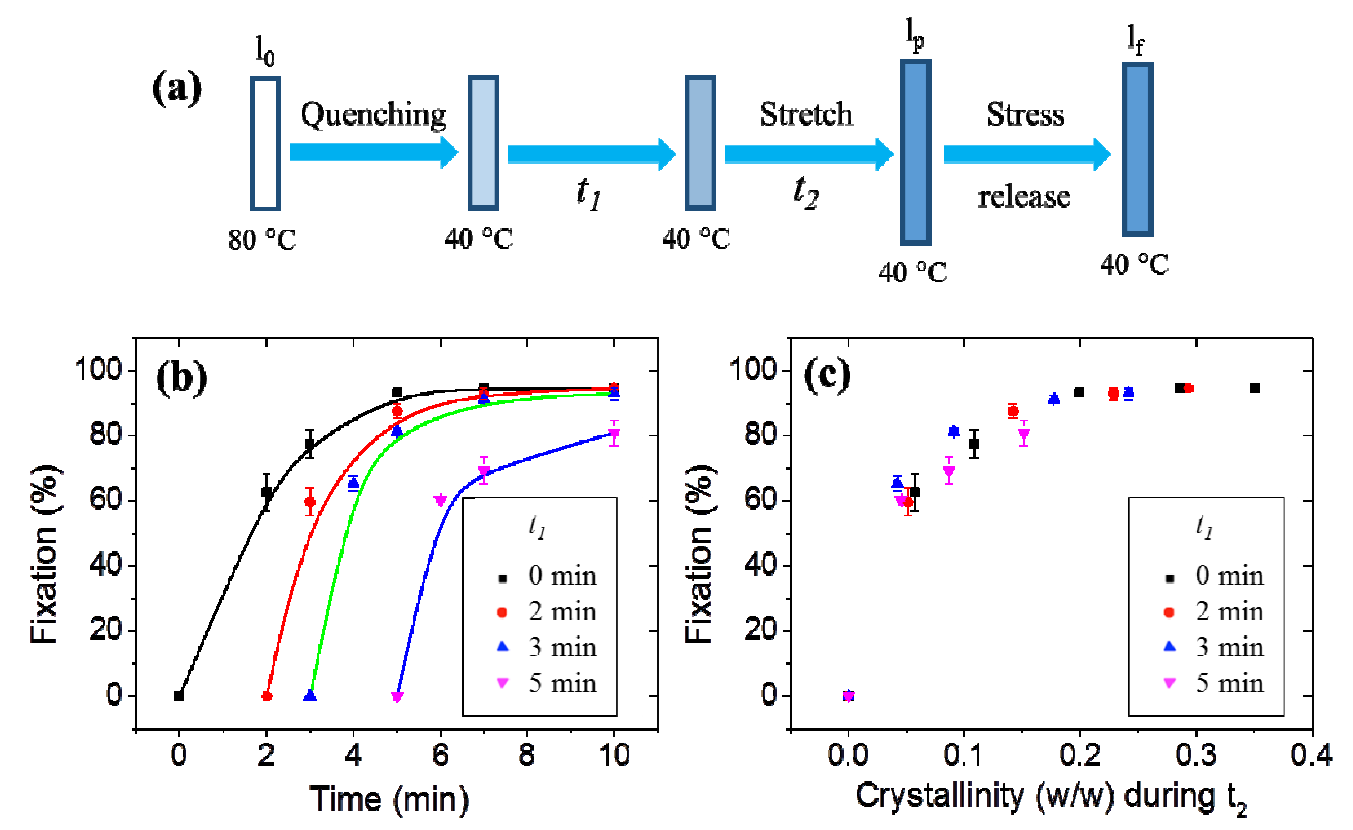

Figure 3: Shape Fixation (a) Shape programming at partial crystallized states (the dark variation of the shape color corresponds to the crystallinity increase). The sample POA of length $l_{0}$ is quickly quenched from the melt $\left(80{ }^{\circ} \mathrm{C}\right)$ to $40{ }^{\circ} \mathrm{C}$ followed by isothermal crystallization. The sample was kept at its initial length $l_{0}$ for time $t_{1}$, and then stretched to a programmed length $l_{p}$ (15\% extension) for time $t_{2}$. Then, the external force was released and the final length $l_{f}$ measured. Fixation is quantified as $F=\left(l_{f}-l_{0}\right) /\left(l_{p}-l_{0}\right)$. (b) After different initial crystallization times $t_{1}(0,2,3,5 \mathrm{~min})$, the fixity parameter $F$ is plotted against total time of crystallization $t=t_{1}+t_{2}$. (c) The fixity parameter is plotted against mass fraction of the crystalline phase formed during $t_{2}$. The existing crystals, which were formed during $t_{1}$, show little effect on the amount of new crystals required for fixation.

\section{Shape Recovery}

In order to undergo TSM, the two programmed shapes must recover at different temperatures upon heating. We used the experiment outlined in Figure $4 \mathrm{a}$ to demonstrate that shapes fixed at different times during isothermal crystallization undergo recovery at different temperatures during subsequent heating. A sample of POA was programmed using the same protocol as in the shape fixation studies outlined in Figure 3a. This was followed by heating the sample at a constant rate of 5 $\mathrm{K} / \mathrm{min}$ in order to monitor the recovery of shapes that were fixed at different stages of crystallization. The crystallization time intervals $t_{1}$ and $t_{2}$ were varied, but the total crystallization time $t_{1}+t_{2}=10 \mathrm{~min}$ was maintained in each case to ensure a 
consistent total crystallinity of $36 \mathrm{w} / \mathrm{w} \%$. Figure $4 \mathrm{~b}$ shows two stages of shape recovery upon heating that depend on the variation of $t_{1}$ and $t_{2}$. The fraction of initial strain relaxation increases for shapes memorized at later stage of crystallization (larger $t_{1}$ ). The sequential shape variation is tentatively assigned to hierarchy of the crystallization process, where nucleation and growth of primary crystals are followed by formation of smaller secondary crystals. As such, there is generally a larger fraction of smaller crystals formed at later stage of isothermal crystallization, which melt at lower temperatures. This hierarchy of sequential crystallization and melting was further proved by AFM and DSC experiments (Supplementary Figures S4, S5).

(a)

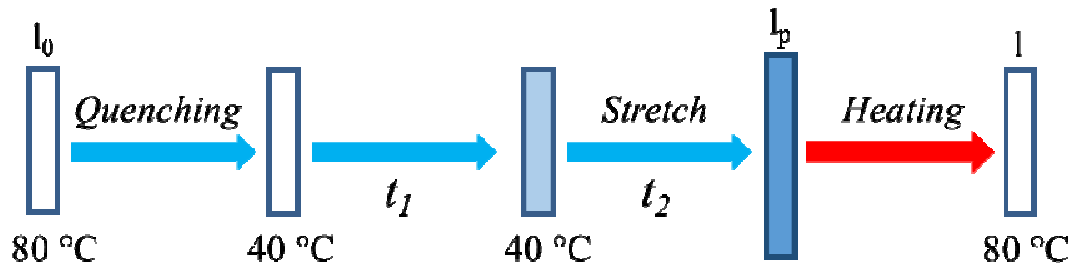

(b)

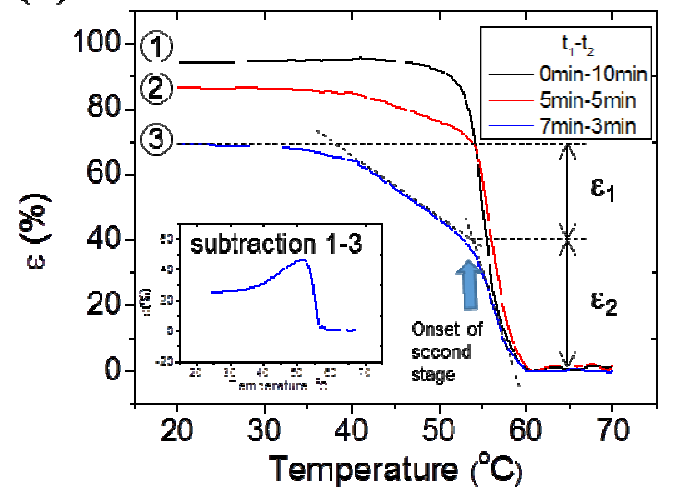

(c)

$40^{\circ} \mathrm{C}$

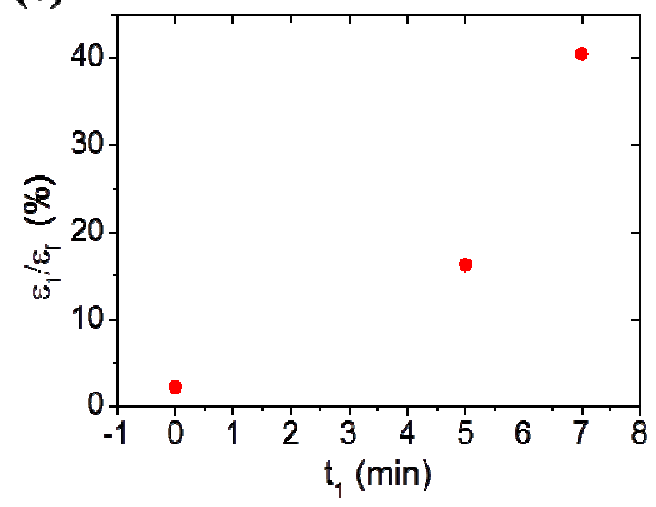

Figure 4: Shape Recovery (a) POA was programmed using the same protocol used for the shape fixation (Figure 3a). Total crystallization time $\left(t_{l}+t_{2}\right)$ was constant as $10 \mathrm{~min}$. After programming, the sample was heated at $5 \mathrm{~K} / \mathrm{min}$, and length $l$ was measured during the shape recovery process. (b) The variation of strain $\varepsilon=\left(l-l_{0}\right) /\left(l_{p}-l_{0}\right)$ during heating is plotted for different allocations of total crystallization time between the annealing time $t_{1}=(0$, $5,7 \mathrm{~min})$ and shape fixation time $t_{2}=(10,5,3 \mathrm{~min})$. Samples 2 and 3 fixed at the later stages of crystallization start their shape recovery at lower temperatures exhibiting a two-stage recovery. The corresponding strain contributions $\left(\varepsilon_{1}\right.$ and $\left.\varepsilon_{2}\right)$ are separated as shown by dashed lines. Inset: Subtracting curve $3\left(t_{1}=7 \mathrm{~min}, t_{2}=3 \mathrm{~min}\right)$ from curve $1\left(t_{1}=0 \mathrm{~min}, t_{2}=\right.$ $10 \mathrm{~min}$ ) shows a hypothetical shape change upon shape programming in two opposite directions during $t_{1}$ and $t_{2}$, respectively. (c) Percentage of strain recovered during first stage of recovery is plotted as fraction of total stored strain $\left(\varepsilon_{1} / \varepsilon_{f}\right)$ against $t_{l}$. 


\section{TSM optimization in One-Way Reversible Shape Memory}

The utility of isothermal programming of shape memory was demonstrated through realization of one-way reversible shape memory transformation - a particular example of TSM. For programming the reversible shape-shifting, we adapted the previously developed protocol [8] to isothermal crystallization. As shown in Figure 5a, a sample of POA was bent from a straight ribbon (shape 1) to a hairpin (shape 2) and back to a straight ribbon (shape $3, \Theta_{1}$ ). In terms of time, the hairpin was programmed during crystallization time $t_{1}$ and then it was re-programmed by reverse deformation to a straight ribbon followed by its annealing during $t_{2}$. Once the crystallization was complete, we released the external force to measure angle $\Theta_{1}$. Upon melting (Figure $5 b$ ), the sample recovered the intermediate hairpin shape (reaching a maximum angle $\Theta_{2}$ at $\left.\sim 58{ }^{\circ} \mathrm{C}\right)$, and then unbent towards the original straight ribbon shape $\left(\Theta_{0}=0\right)$ as the temperature reached $62{ }^{\circ} \mathrm{C}$. This experiment shows that two confronting shapes, such as ribbon and hairpin, may be separately memorized by different fractions of the crystalline scaffold formed at different stages of isothermal crystallization. This enabled temperature separation of the oppositely directed shape transformations upon heating, when the shape secured by the later-formed crystallites (shape 3) starts its recovery to shape 2 at lower temperatures. Since shape 2 was secured by a larger fraction of primary crystals, the recovery of shape 1 occurs at higher temperatures, which resulted in apparent reversibility of the shape transformation. Note that this reversible shape-shifting was expected from our prediction of the conflict between programming of oppositely directed shapes (inset in Figure 4b). The same protocol can be applied to copolymers at different temperature range, with similar TSM behavior (Supplementary Figure S6).

The extent of the angle change upon heating may be tuned by controlling the durations $t_{1}$ and $t_{2}$, and thus varying the fraction of crystallites utilized to encode each shape. While crystallization times $t_{1}$ and $t_{2}$ were varied, the total crystallization time $t_{1}+t_{2}=10 \mathrm{~min}$ was maintained the same to ensure a 
consistent total crystallinity. A series of samples were isothermally programmed with different sets of $t_{1}$ and $t_{2}$ to measure the angle change from $\Theta_{1}$ to $\Theta_{2}$ as shown in Figure 5c. In each case, $\Theta_{2}$ was maximized upon heating to $58^{\circ} \mathrm{C}$, which corresponds to melting of $26 \mathrm{w} . \%$ of crystals. As $t_{1}$ increased, fixation for the second shape was more difficult due to the high crystallinity acquired during $t_{1}$. Although this resulted in a lower fixity ratio of the final shape (larger $\Theta_{1}$ ), we observed much better restoration of the intermediate hairpin shape (larger $\Theta_{2}$ ). By subtracting $\Theta_{1}$ from $\Theta_{2}$, we show that maximum reversibility is achieved at $t_{1} \cong 2 \mathrm{~min}$, which corresponds to a crystallinity of $\sim 5 \%$ (Figure $5 \mathrm{~d}$ ). This behavior can be explained by the interplay of crystals required to fix the first and second shapes, respectively. When the initial fixation time $\left(t_{1}\right)$ was too short, there were not enough crystals to memorize the first programmed shape, resulting in smaller $\Theta_{2}$. However, a longer $t_{1}$ results in higher crystallinity, which may hinder deformability of the sample and allow insufficient amount of crystals for the second fixation resulting in an increase of $\Theta_{1}$. By optimizing the time portioning between the two stages of crystallization, we have achieved a maximum reversibility within an angle range of 60 degrees. 
(a)

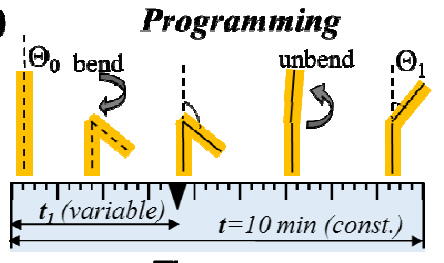

(b)

Time

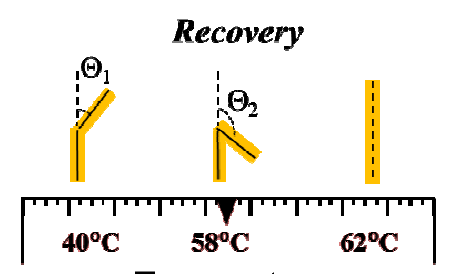

Temperature

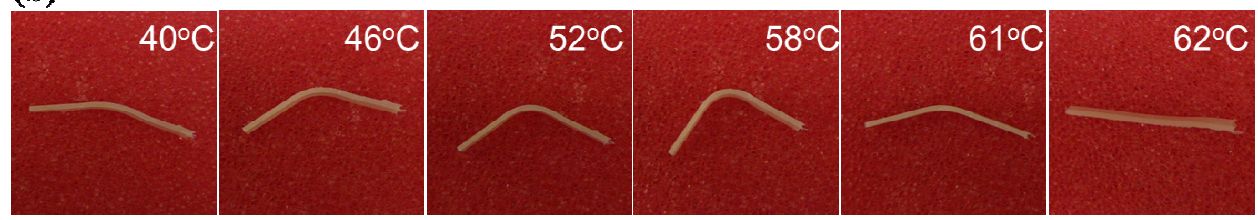

Heating

(c)

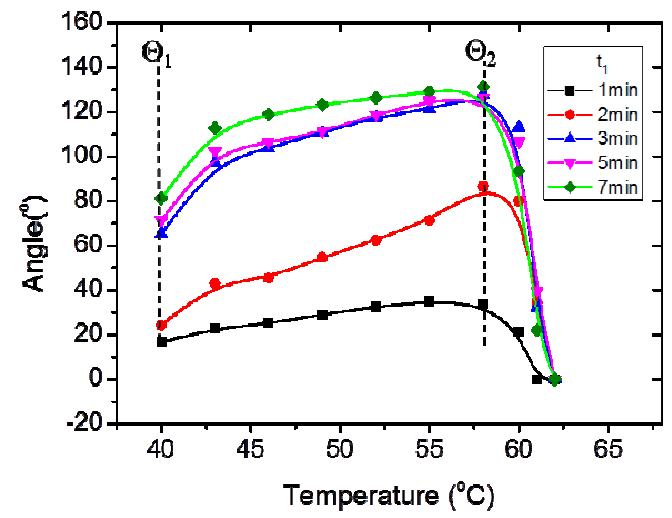

(d)

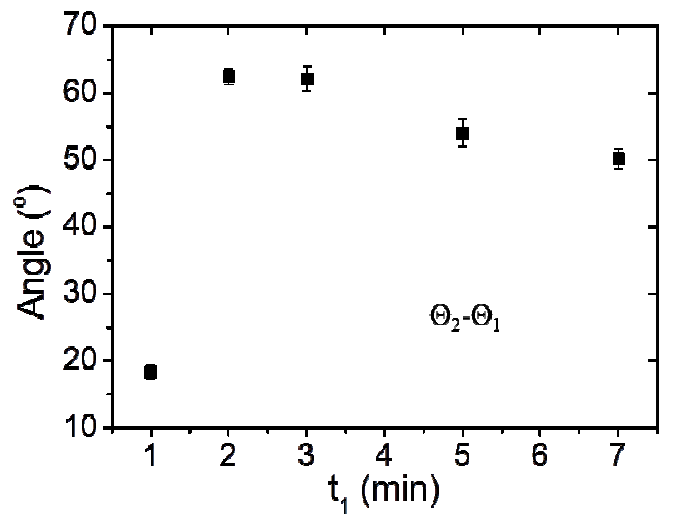

Figure 5: TSM Applications in One-Way Reversible Shape Memory (a) Programming and recovery protocols for one-way reversibility. A sample of POA was cooled to $40{ }^{\circ} \mathrm{C}$ and bent $180^{\circ}$. After time $t_{1}$, the sample was unbent to a straight ribbon shape for time $t_{2}$ until $t_{1}$ $+t_{2}=10$ min was reached. The angle of the sample after programming was measured as $\Theta_{1}$. Upon heating, the sample bends and then unbends through heating. The maximum angle occurs ca. $58{ }^{\circ} \mathrm{C}$ and is recorded as $\Theta_{2}$. (b) Images of a one-way reversible shape change from heating through $40-62{ }^{\circ} \mathrm{C}$. The sample was programmed isothermally at $40{ }^{\circ} \mathrm{C}$ using the protocol described in the Experimental Section with $t_{1}=2 \mathrm{~min}$. (c) Variation in shape change during recovery with variation of $t_{1}$. Angle change shown for $t_{1}=1,2,3,5$ and $7 \mathrm{~min}$. Angles $\Theta_{1}$ and $\Theta_{2}$ are determined at the corresponding intersections with the dashed lines. Samples with short $t_{1}$ have little memory of the second shape, resulting in small $\Theta_{2}$. Long $t_{1}$ intervals hamper the fixation of the third shape, resulting in large $\Theta_{1}$. (d) The angle change during the recovery process was measured as the difference between $\Theta_{1}$ and $\Theta_{2}$. Reversibility can be controlled as a function of $t_{l}$ and maximized ca. $t_{1}=2 \mathrm{~min}$. 


\section{CONCLUSION}

We have introduced a new method for TSM programming, which can be accomplished isothermally without the requirement of advanced materials with unusual transition temperatures. With new protocols we can perform a variety of different TSM transformations including one-way reversible shape memory. Crystallization time can be used to control and optimize the shape memory process and observed phenomenon might be used to relate the role of microscopic crystallites in macroscopic shape changes in future shape memory studies.

\section{ASSOCIATED CONTENT}

\section{Supporting information}

Detailed properties of characterized materials; DSC and WAXS analysis on crystallization kinetics; strain effect on materials; AFM and DSC study on sequential melting.

ACKNOWLEDGMENTS: The authors acknowledge financial support from the National Science Foundation DMR-1122483, DMR-1004576, DMR-1206957, and DMR-1436201. Research carried out at the Center for Functional Nanomaterials (CFN) and National Synchrotron Light Source (NSLS) at the Brookhaven National Laboratory has been supported by the U.S. Department of Energy, Office of Basic Energy Sciences, under Contract DE-AC02-98CH10886. The authors thank O. Gang and D. Nykypanchuk for the help with WAXS analysis.

AUTHORS CONTRIBUTION: J.Z. composed the manuscript and performed the experiments, S.A.T. and Q.L. synthesized materials and helped with writing, V.S.A. discussed results and contributed to revisions, S.S.S. supervised the project. 
NOTES: The authors declare no competing financial interests.

\section{REFERENCES}

1. Lendlein A, Langer R. Science 2002;296(5573):1673-1676.

2. Mather PT, Luo XF. ACS Macro Letters 2013; 2: 152-156.

3. Yu YL, Nakano M, Ikeda T. Nature 2003;425(6954):145-145.

4. Li G, Fei GX, Xia HS, Han JJ, Zhao Y. Journal of Materials Chemistry 2012;22(16):7692-7696.

5. Wu X, Huang WM, Zhao Y, Ding Z, Tang C, Zhang J. Polymers 2013; 5:1169-1202

6. $\quad$ Liu, Y., Boyles, J. K., Genzer, J., Dickey, M. D. Soft Matter 2012; 8(6):1764-1769.

7. Mondal S. Mini-Reviews in Organic Chemistry 2009;6(2):114-119.

8. Zhou J, Turner SA, Brosnan SM, Li Q, Carrillo J-M, Nykypanchuk D, Gang O, Ashby, VS, Dobrynin A, Sheiko SS. Macromolecules 2014;47(5):1768-1776.

9. Behl M, Lendlein A. Journal of Materials Chemistry 2010;20(17):3335-3345.

10. Luo YW, Guo YL, Gao X, Li BG, Xie T. Advanced Materials 2013;25(5):743-748.

11. Meng H, Li GQ. Polymer 2013;54(9):2199-2221.

12. Bellin I, Kelch S, Langer R, Lendlein A. Proceedings of the National Academy of Sciences of the United States of America 2006;103(48):18043-18047.

13. Xie T, Xiao XC, Cheng YT. Macromolecular Rapid Communications 2009;30(21):1823-1827.

14. Xie T. Nature 2010;464(7286):267-270.

15. Yu K, Xie T, Leng JS, Ding YF, Qi HJ. Soft Matter 2012;8(20):5687-5695.

16. Behl M, Kratz K, Noechel U, Sauter T, Lendlein A. Proceedings of the National Academy of Sciences of the United States of America 2013;110(31):12555-12559.

17. Kratz K, Madbouly SA, Wagermaier W, Lendlein A. Advanced Materials 2011;23(35):4058-4062.

18. Kratz K, Voigt U, Lendlein A. Advanced Functional Materials 2012;22(14):3057-3065.

19. Andrews RD, Tobolsky AV, Hanson EE. Journal of Applied Physics 1946;17(5):352-361.

20. Hsiao BS, Gardner KH, Wu DQ, Chu B. Polymer 1993;34(19):3996-4003.

21. Hsiao BS, Wang ZG, Yeh FJ, Gao Y, Sheth KC. Polymer 1999;40(12):3515-3523.

22. Guo L, Spegazzini N, Sato H, Hashimoto T, Masunaga H, Sasaki S, Takata M, Ozaki Y. Macromolecules 2012;45(1):313-328. 
23. Avila-Orta CA, Medellin-Rodriguez FJ, Wang ZG, Navarro-Rodriguez D, Hsiao BS, Yeh FJ. Polymer 2003;44(5):1527-1535.

24. Turner SA, Zhou J, Sheiko SS, Ashby VS. ACS Applied Materials \& Interfaces 2014; 6(11): 8017-8021.

25. Brosnan SM, Brown AH, Ashby VS. Journal of the American Chemical Society 2013;135(8):3067-3072. 


\section{Isothermal programming of triple shape memory}

Jing Zhou, Qiaoxi Li, Sara A. Turner, Valerie Sheares Ashby, and Sergei S. Sheiko*

Department of Chemistry, University of North Carolina at Chapel Hill, NC

27599-3290

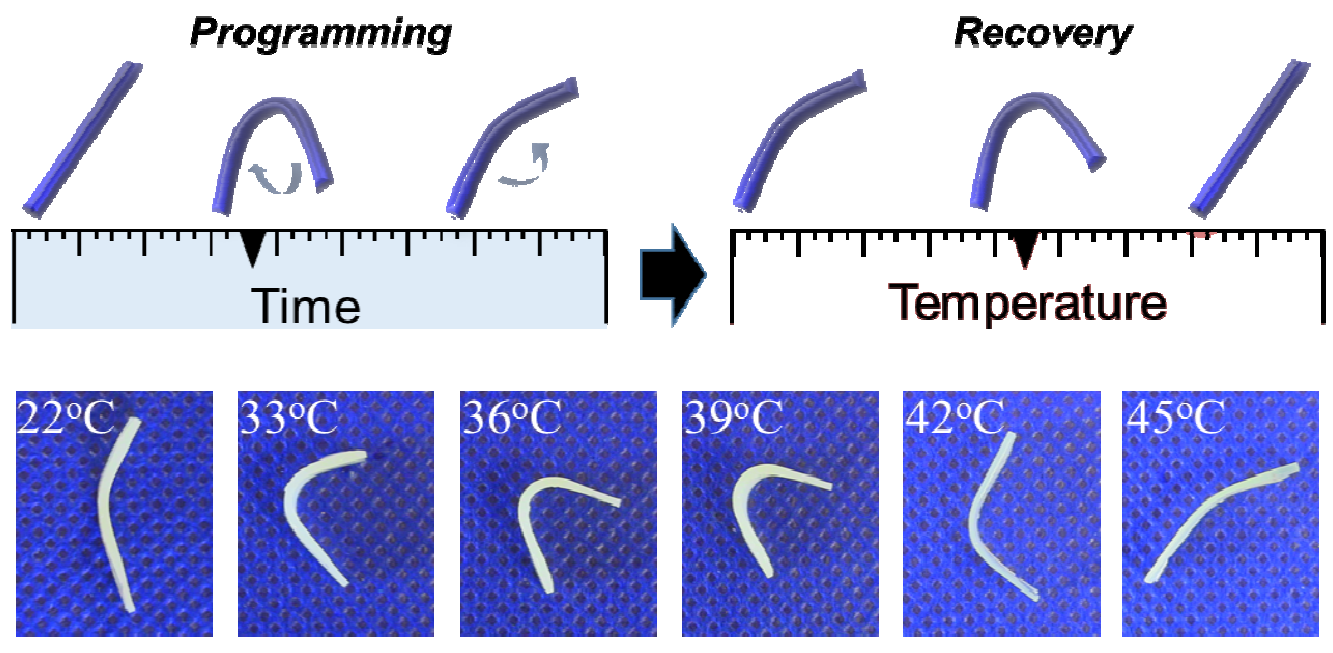

for Table of Contents use only 